\title{
Knockdown of prion protein (PrP) by RNA interference weakens the protective activity of wild-type PrP against copper ion and antagonizes the cytotoxicity of fCJD-associated PrP mutants in cultured cells
}

\author{
ZHAO-YUN WANG, CHAN TIAN, YUAN-YUAN JING, HAN-SHI GONG, YAN GUO, QI SHI, \\ CAO CHEN, SHU-YING ZHU and XIAO-PING DONG
}

\begin{abstract}
State Key Laboratory for Infectious Disease Prevention and Control, National Institute for Viral Disease Control and Prevention, Chinese Center for Disease Control and Prevention, Beijing 102206, P.R. China
\end{abstract}

Received February 22, 2011; Accepted April 1, 2011

DOI: $10.3892 / \mathrm{ijmm} .2011 .688$

\begin{abstract}
Development of the pathogenesis of transmissible spongiform encephalopathies (TSEs) requires the presence of both the normal host prion protein $\left(\mathrm{PrP}^{\mathrm{C}}\right)$ and the abnormal pathological proteinase-K resistant isoform $\left(\mathrm{PrP}^{\mathrm{Sc}}\right)$. Reduction of $\operatorname{PrP}^{\mathrm{C}}$ levels has been shown to extend survival time after prion infection. In this report, based on analysis of the known sequences of human PrP, we constructed two small interfering RNA (siRNA) duplexes targeting the segments of amino acids (aa) 108-114 (Ri2) and aa 171-177 (Ri3). Western blot analysis results revealed that these PrP-specific siRNAs could effectively knock down the levels of either endogenous PrP in human neuroblastoma SHSY-5Y cells or recombinant PrP transfected with the plasmid expressing the full-length human PrP in human embryonic kidney (HEK) 293T cells. Meanwhile, the two siRNAs also showed a significant effect on the reduction of the expression of the PrP-PG9 and PrP-PG12 familial Creutzfeldt-Jakob disease (CJD)-associated PrP mutants with four and seven extra octarepeats, in the cells transfected with the respective expression plasmids. MTT tests identified that knockdown of wild-type PrP by Ri2 and Ri3 did not change the cell growth capacities, but significantly decreased the cell resistances against the challenge of $\mathrm{Cu}^{2+}$. Co-expression of Ri2 and Ri3 partially antagonized the cytotoxicity caused by expressing PrP-PG9 and PrP-PG12 in the two cell lines. Moreover, the rescuing effectiveness of PrP siRNAs was time-related, with the more efficient antagonism of the
\end{abstract}

Correspondence to: Dr Xiao-Ping Dong, State Key Laboratory for Infectious Disease Prevention and Control, National Institute for Viral Disease Control and Prevention, Chinese Center for Disease Control and Prevention, Chang-Bai Rd 155, Beijing 102206, P.R. China E-mail: dongxp238@sina.com

Key words: prion protein, small interfering RNA, Creutzfeldt-Jakob disease, cytotoxicity, copper cytotoxicity of fCJD-associated PrP mutants occurring at the early stages after transfection. The data shown here provide useful clues for seeking potential therapeutic tools for prion diseases.

\section{Introduction}

Prion diseases also known as transmissible spongiform encephalopathies (TSEs) are a group of transmissible neurodegenerative disorders causing severe spongiform degeneration and neuronal loss in the central nervous system (CNS) that could spread though both human and animal contact, such as Creutzfeldt-Jakob disease (CJD) in human, scrapie in sheep and goat, and bovine spongiform encephalopathy (BSE) in cattle $(1,2)$. The commonly accepted view is that the central event in the pathogenesis is the conversion of a host-derived cellular prion protein $\left(\mathrm{PrP}^{\mathrm{C}}\right)$ to an infectious isoform called disease-associated scrapie prion protein $\left(\mathrm{PrP}^{\mathrm{Sc}}\right)$, which is the insoluble, misfolded and proteinase K (PK)-resistant form accumulating in the CNS (3-6). Propagation and accumulation of infectious PrP have always been thought to be tightly linked to the pathogenesis of prion disease. Host $\operatorname{PrP}^{\mathrm{C}}$ is essential for prion propagation and pathogenesis, since no prion replication occurs in mice devoid of $\operatorname{PrP}^{\mathrm{C}}$.

Prion disease is fatal without available specific therapeutic methodology. Because serious damage to the brain has already occurred before clinical symptoms manifest, potential treatments for prion disease so far have aimed not to cure the disease, but to slow disease progression (7). Considering the important role of PrP in prion disease, two basic therapeutic strategies have been adopted: reduction of $\mathrm{PrP}^{\mathrm{C}}$ levels and prevention of the conversion from $\operatorname{PrP}^{\mathrm{C}}$ to $\mathrm{PrP}^{\mathrm{Sc}}$ (8). It has been demonstrated that mice devoid of $\mathrm{PrP}^{\mathrm{C}}$ are resistant to scrapie and fail to propagate prions and that the re-introduction of the PrP-encoding gene restores susceptibility to the disease. These findings highlight that the establishment of prion disease-resistance by knocking down the PrP gene using RNA interference (RNAi) technology will be a potential therapeutic tool for prion disease (9-12). 
RNAi is an evolutionarily highly conserved, sequencespecific post-transcriptional gene-silencing mechanism, whereby small interfering RNA (siRNA) targets homologous mRNA for degradation (13). siRNAs are generated from endogenous or exogenous double-stranded RNAs (dsRNAs) by the dsRNA endonuclease, dicer; subsequently, siRNA activates the RNA-induced silencing complex, RISC, to degrade the target mRNAs (14). A single administration of lentivirus expressing a short hairpin RNA (shRNA) targeting PrP into the hippocampus of mice with established prion disease significantly prolonged survival time (15). Lentiviral-mediated RNAi of PrP also prevented the onset of behavioral deficits associated with early prion disease, reduced spongiform degeneration, and protected against neuronal loss (16). Introduction of siRNAs targeting the PrP gene transcripts suppressed $\mathrm{PrP}^{\mathrm{Sc}}$ formation and ultimately eliminated $\mathrm{PrP}^{\mathrm{Sc}}$ from prioninfected cell lines (17).

$\operatorname{PrP}^{\mathrm{C}}$ is believed to be able to act as a copper ion buffer protecting cells from damage of the unfixed $\mathrm{Cu}^{2+}(18,19)$. Neurons containing PrP are more resistant to copper toxicity than cells lacking PrP (20). Some sorts of PrP knockout mice show extensive oxidative damage that is not present in wildtypes (21-23). On the other hand, familial CJD (fCJD)-associated PrP mutants, including point-mutations and octarepeat insertions, reveal obvious cytotoxicity (24), even induce experimental TSEs in transgenic mice (25). To get insight into the physiological activity of $\mathrm{PrP}^{\mathrm{C}}$ and seek potential interference on the cytotoxicity, even pathogenesis induced by fCJD-associated PrP mutants, two PrP specific siRNAs were synthesized and their abilities to knockdown either endogenous or exogenous PrP levels in cultured cells were confirmed. In the presence of PrP specific siRNAs, the protective effect of wild-type $\mathrm{PrP}$ against $\mathrm{Cu}^{2+}$-induced cytotoxicity were decreased, while the cytotoxicities caused by gCJD mutated PrPs were reversed.

\section{Materials and methods}

Plasmid construction. To construct the recombinant plasmids expressing siRNA, the siLent Gene ${ }^{\mathrm{TM}}$ U6 Cassette RNA Interference system (Promega, USA) was used. Based on the analyses of the known sequences of human PrP available on the internet (Ambion, USA), two conservative motifs were identified as the potential targeting areas for PrP-specific siRNAs: 5'-AACATGAAGCACATGGCTGGT-3', (nt GATC CAACATGAAGCACATGGCTGGTTTCAAGAGAACCAG CCATGTGCTTCATGTTTTTTGGAAA) and 5'-AACCAG AACAACTTTGTGCAC-3', (nt GATCCGCCAGAACAACT TTGTGCACTTCAAGAGAGTGCACAAAGTTGTTC TGGTTTTTTGGAAA), with BamHI and HindIII restriction sites at each terminus. The sequences transcribing the PrP siRNAs were chemically synthesized (Sangon, Shanghai, China) as a 21 bp-long inverse repeat separated by a 9 bp loop for each sequence. After denaturing and annealing at room temperature, the complementary double-strand sequences were inserted into the downstream region of the U6 promoter within the vector pSilence2.1, generating the plasmids, pPrPRi2 and pPrP-Ri3. The plasmids, pcDNA3.1-PrP-PG5 expressing the full-length wild-type (WT) human PrP (aa 1-253) with 5 octarepeats (PG5) (26), pcDNA3.1-PrP-PG9 expressing the human PrP with 4 extra octarepeats (PG9) and the pcDNA3.1-PrP-PG12 with 7 extra octarepeats had been previously generated (27).

Cell culture and transfection. The human neuroblastoma SHSY-5Y cells with detectable endogenous PrP protein and the human embryonic kidney (HEK) 293T cells without detectable endogenous PrP protein were maintained in Dulbecco's modified Eagle's medium (DMEM, Gibco-BRL, USA) supplemented with $10 \%$ (v/v) fetal cattle serum (FCS). Cells at the logarithmic growth phase were plated into 6-well plates (Falcon, USA) $24 \mathrm{~h}$ before transfection. Plasmid DNA (2 $\mu$ g each) was transiently transfected with Fugene ${ }^{\mathrm{TM}}$ reagent (Roche, Switzerland), according to the manufacturer's instructions. Forty-eight hours after transfection, cells were harvested and employed in further experiments.

Cell viability assays. The cultured cells at the logarithmic growth phase were trypsinized and seeded on a 96-well plate at a concentration of $10^{4}$ cells/well and transiently transfected with individual recombinant plasmids by the Fugene ${ }^{\mathrm{TM}}$ reagent. Twelve hours post-transfection, the transfected cells were challenged with $60 \mu \mathrm{M} \mathrm{Cu}^{2+}$ for $12 \mathrm{~h}$ or $5 \mathrm{mM} \mathrm{H}_{2} \mathrm{O}_{2}$ for $5 \mathrm{~min}$ or $10 \mu \mathrm{M}$ colchicine for $12 \mathrm{~h}$. Afterwards, the culture medium was changed with regular DMEM medium and the cell viability was determined with an MTT assay by assessing the conversion of 3-(4,5-dimethylthiazol-2-yl)-2,5-diphenyl-2Htetrazolium bromide (MTT, Sigma, USA) to a formazan product. The reaction was terminated by removal of the supernatant and addition of $200 \mu \mathrm{l} \mathrm{DMSO} /$ well to dissolve the formazan product. Absorbance was measured at $492 \mathrm{~nm}$ in a spectrophotometer. Each assay was performed in duplicates of at least four wells.

Western blot analyses. The cellular lysates were separated by $12 \%$ SDS-PAGE and electrotransferred onto nitrocellulose membranes. After blocking with $5 \%$ nonfat-dried milk in PBST (phosphate-buffered saline, $\mathrm{pH} 7.6$, containing $0.05 \%$ Tween-20) for $1 \mathrm{~h}$ at room temperature, the membranes were incubated with 1:4,000 PrP-specific monoclonal antibody (mAb) 3F4 (Dako, Denmark), 1:2,000 diluted mAb antihuman $\beta$-actin (Santa Cruz Biotechnology, USA) for $2 \mathrm{~h}$ at room temperature, and then incubated with 1:10,000 diluted horseradish peroxidase (HP)-conjugated anti-mouse IgG (Santa Cruz Biotechnology). The specific signals were visualized by the ECL kit (PE Applied Biosystems, Foster City, USA).

Statistical analyses. Quantitative analysis of immunoblotting images was carried out using the computer-assisted software Image Total Tech (GE Healthcare, USA). The values of each target blot were evaluated. All data are presented as the mean \pm SD. Statistical analysis was performed using the t-test. Probabilities of $<0.05$ were considered to indicate statistical significance. Statistical analysis of the MTT assay was performed using the SPSS (version 17.0) and the data are presented as mean \pm SD. One-way ANOVA was used to compare the differences in the OD 492 values between the transfected and the vector control groups. A P-value $<0.05$ was considered to indicate statistical significance. 
A

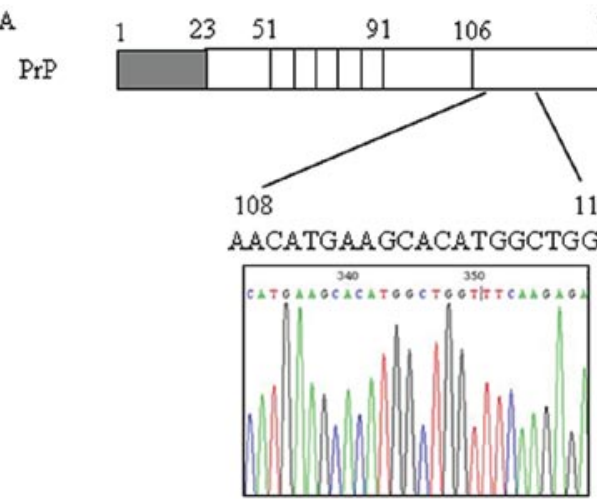

RNAi2

B
SHSY-5Y

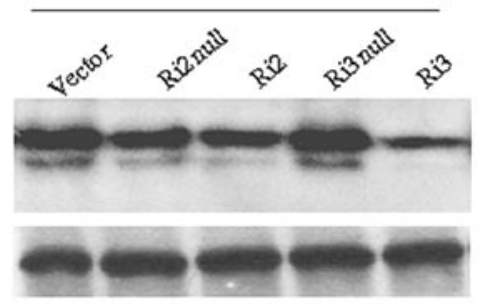

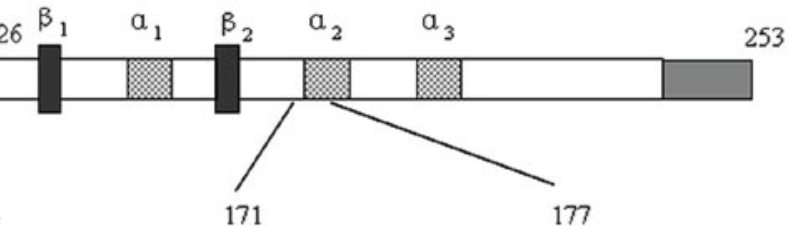

AACCAGAACAACTTTGTGCAC

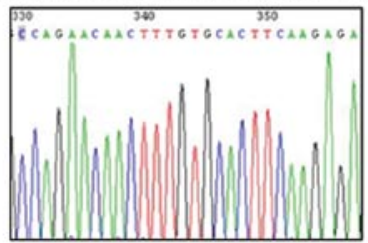

RNAi3
$\operatorname{PrP}$

$\beta$-actin

Figure 1. Knockdown of the expression levels of cellular PrPs by two PrP-specific siRNAs. (A) The targeting sites and sequences of PrP-specific siRNAs Ri2 and Ri3. The upper part is the diagram of the full-length human $\operatorname{PrP}$ (aa 1-253), with the N-terminal signal peptide, five octarepeats, two $\beta$-sheets, three $\alpha$-helixes and the C-terminal GPI anchor. The lower part shows the nucleotide sequences and the individual targeting regions in PrP of Ri2 and Ri3. (B) The inhibiting effects of Ri2 and Ri3 on the expression of endogenous PrP in SHSY-5Y cells (left panel) or recombinant PrP transfected with a plasmid expressing full-length WT human PrP in 293T cells (right panel) by a PrP-specific Western blot analysis. Various transfected RNAi plasmids are showed on the top and the specific signals of the proteins are indicated on the right.

\section{Results}

Inhibition of the expression of endogenous PrP in SHSY-5Y cells and transfected PrP in 2937 cells. To generate human PrP-specific siRNA, two 21 bp-long sequences were synthesized, which targeted the hydrophobic region (aa 108-114, Ri2) and the second $\alpha$-helix (aa 171-177, Ri3), respectively (Fig. 1A). Meanwhile, two 21-bp segments with same compositions of nucleosides as Ri2 or Ri3, but with randomly arrayed sequences, were synthesized, and named Ri2null and Ri3null, respectively. Individual double-stranded segments were inserted into the vector pSilence2.1, generating respective transcribing plasmids.

To evaluate the inhibitory effects of the constructed PrP-specific siRNA plasmids on the expression of endogenous PrP, various plasmids were transiently transfected into SHSY-5Y cells. Western blot analyses showed weaker PrP signals in the preparations receiving the plasmids transcribing Ri2 (Fig. 1B, left panel, lane 3) and Ri3 (lane 5), compared with that of the cells receiving the vector (lane 1), whereas the intensities of PrP signals in the cells expressing Ri2null (lane 2) and Ri3null (lane 4) remained almost unchanged. To evaluate the influences of the PrP-specific siRNA on the expressing levels of the recombinant PrP in cells, 293T cells were co-transfected with pcDNA3.1-PG5 expressing WT human full-length PrP and the PrP-specific siRNA plasmids. As expected, a PrP-specific immunoblot revealed a weak PrP-signal in the preparation of Ri2 (Fig. 1B, right panel, lane 3) and almost no signal in that of Ri3 (lane 5), while the PrP signals in the Ri2null and
Ri3null preparations remained unchanged (lanes 2 and 4). The results indicate that both PrP-specific siRNAs, Ri2 and Ri3, can significantly inhibit the expression of both endogenous PrP in SHSY-5Y cells and of transfected PrP in 293T cells.

To assess the inhibitory capacities of PrP-specific siRNAs on the expression of cellular PrP, different amounts of siRNA expressing plasmids $(0,0.25,0.5$ and $1 \mu \mathrm{g})$ were transfected into SHSY-5Y cells or co-transfected into 293T cells together with $1 \mu \mathrm{g}$ pcDNA3.1-PG5. Forty-eight hours post-transfection, the cells were harvested and the levels of cellular PrP were tested by Western blot analyses. The results demonstrate that in both cell lines, the signal intensities of cellular PrP gradually decreased along with increases of the transfected siRNA plasmids (Fig. 2A), showing a dose-dependent alteration. Quantitative measures of the gray value of each immunoblot vs. that of $\beta$-actin showed the similar repressive tendencies of siRNAs, Ri2 and Ri3 on the expression of cellular PrP in those two cell lines, while Ri3 seemed to have relatively stronger repression effectiveness on the expression of $\operatorname{PrP}$ in SHSY-5Y cells than Ri2 (Fig. 2B).

Expression of PrP-specific siRNA reduces the cell protective activity of wild-type human PrP against the challenge of copper. Cellular $\operatorname{PrP}^{\mathrm{C}}$ has shown a protective function on the cytotoxicity induced by some chemical elements, such as $\mathrm{Cu}^{2+}$ (28). To evaluate the potential influence of PrP-specific siRNAs on cell growth, plasmids expressing Ri2 and Ri3 were separately introduced into SHSY-5Y cells or co-transfected 
A.

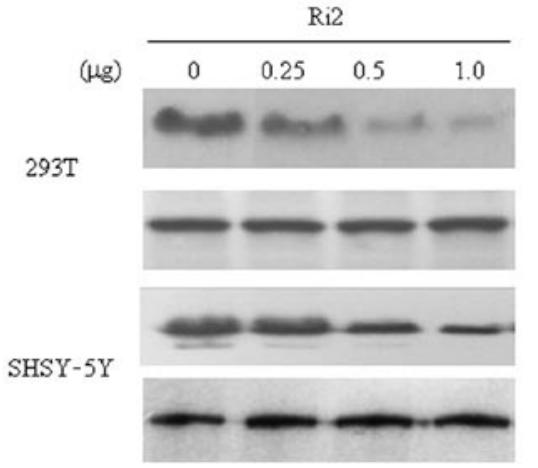

B

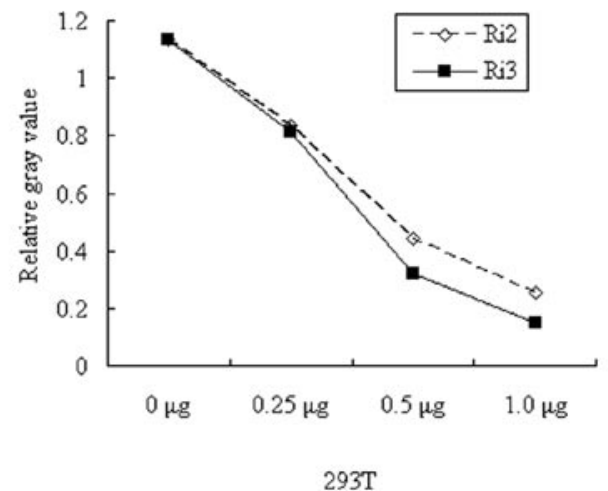

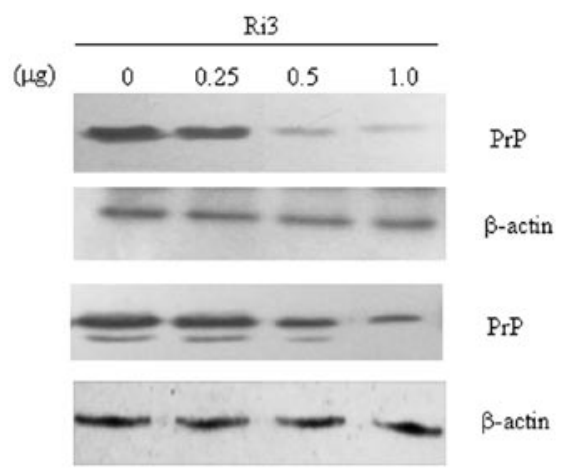

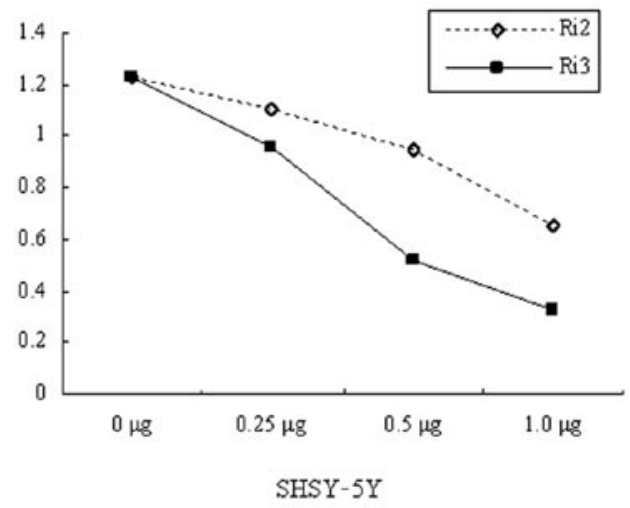

Figure 2. The interfering efficacy assays of two siRNAs on the levels of PrP in 293T and SHSY-5Y cells. (A) Western blot analyses. Different amounts of siRNA expressing plasmids $(0,0.25,0.5$ and $1 \mu \mathrm{g})$ were transfected into SHSY-5Y cells or co-transfected together with $1 \mu \mathrm{g}$ pcDNA3.1-PG5 into the 293T or SHSY-5Y cell lines. Cells were harvested $48 \mathrm{~h}$ post-transfection. (B) Quantitative measures of the gray value of each immunoblot vs. that of $\beta$-actin.

A

$293 \mathrm{~T}$

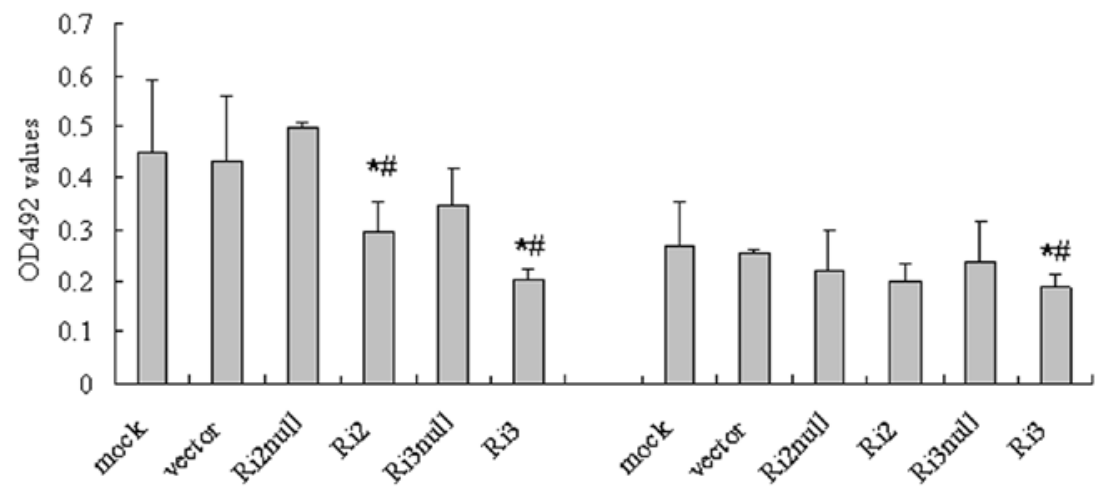

B

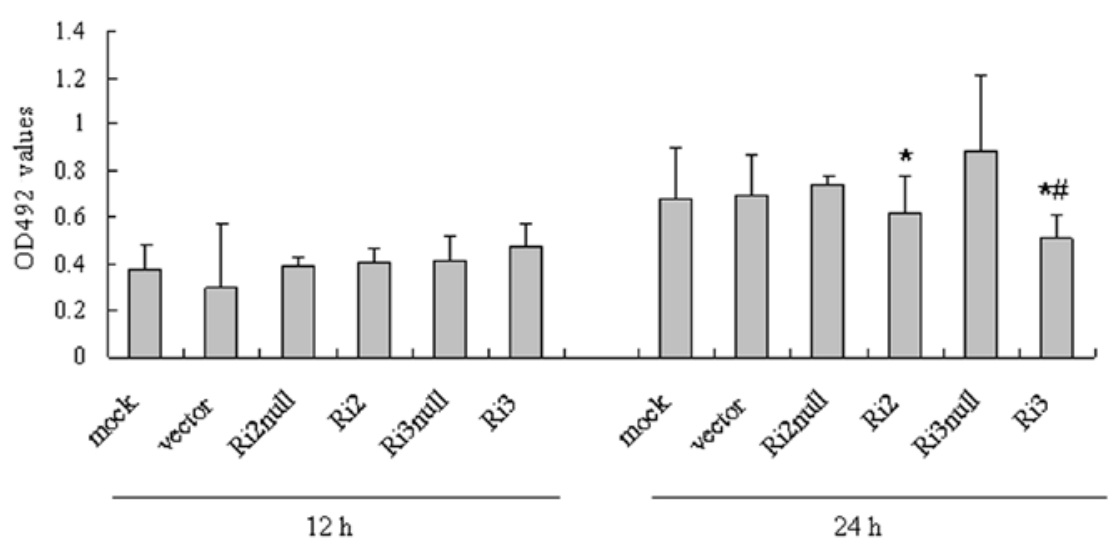

Figure 3. MTT assays for the inhibition of the siRNAs on the protective activities of cellular $\operatorname{PrP}^{\mathrm{C}}$ against the challenge of $\mathrm{Cu}^{2+}$. Cells were treated with $60 \mu \mathrm{M}$ of $\mathrm{Cu}^{2+}$ at $12 \mathrm{~h}$ (left panel) or $24 \mathrm{~h}$ (right panel) post-transfection and MTT tests were performed $12 \mathrm{~h}$ after $\mathrm{Cu}^{2+}$ challenge. (A) 293T cells. (B) SHSY-5Y cells. The average data of each preparation was calculated based on three independent experiments and are represented as the mean \pm SD. "Statistical difference $(\mathrm{P}<0.05)$ compared with the data of the respective Ri-null; ${ }^{\#}$ statistical difference $(\mathrm{P}<0.05)$ compared with the data of the mock control. 

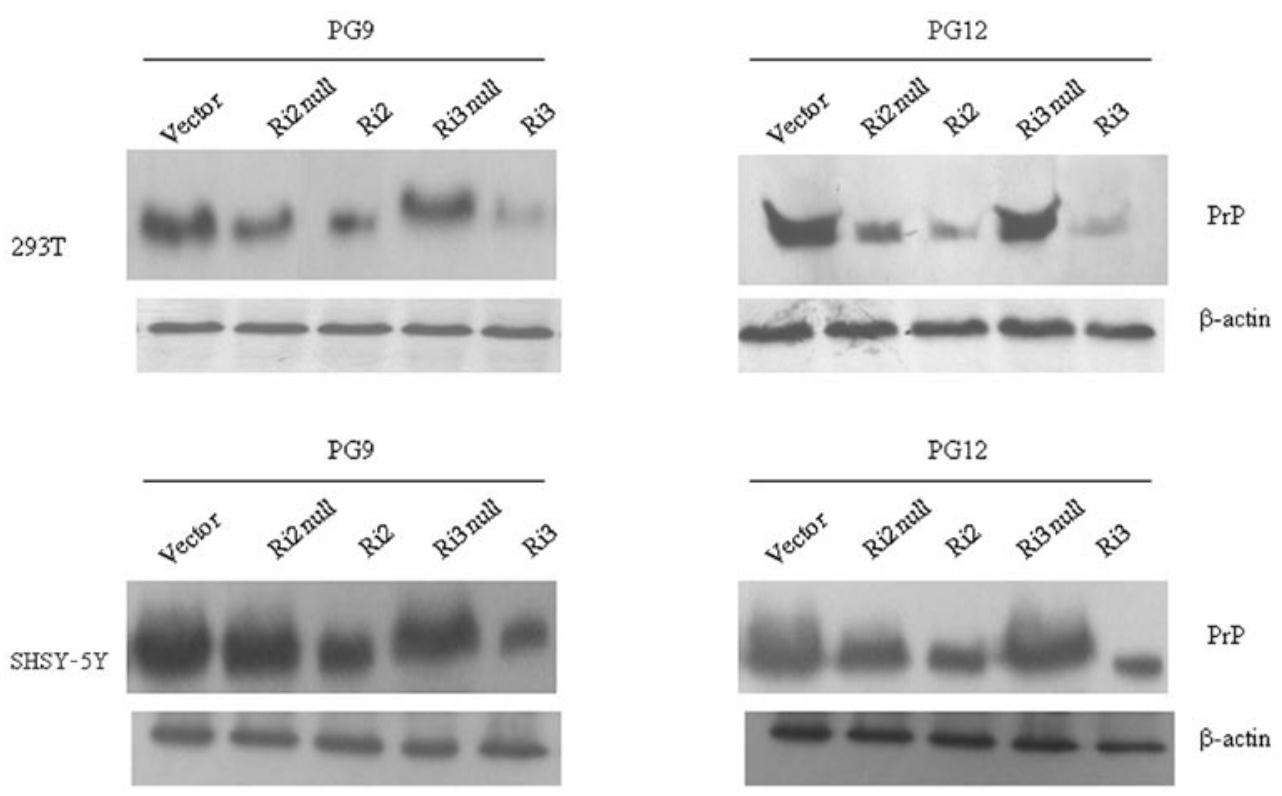

Figure 4. The inhibitory effects of Ri2 and Ri3 on the expression of recombinant PrP mutants in the cultured cells expressing insertional mutated human PrP-PG9 (left panel) and -PG12 (right panel) by PrP-specific Western blot analyses. (A) 293T cells. (B) SHSY-5Y cells. Various transfected RNAi plasmids are shown on the top and the specific signals of the proteins are indicated on the right.

into 293T cells together with pcDNA3.1-PG5. MTT assays did not reveal a significant difference in the cell viabilities between the cells expressing the PrP-specific siRNAs and those not in either of the two cell lines examined (data not shown). To evaluate whether knockdown of cellular PrP can change the cytotoxicity of copper, cultured cells were treated with $60 \mu \mathrm{M}$ $\mathrm{Cu}^{2+}$ at 12 (Fig. 3, left panel) or $24 \mathrm{~h}$ (Fig. 3, right panel) posttransfection and MTT tests were performed $12 \mathrm{~h}$ after $\mathrm{Cu}^{2+}$ challenge. In 293T cells transfected with pcDNA3.1-PG5 beforehand, co-transfections of Ri2 and Ri3 expressing plasmids resulted in reductions of cell viabilities, whereas co-transfections of Ri2null- and Ri3null-expressing plasmids did not affect the cell viabilities compared with that of cells co-transfected with blank vector or mock preparation (Fig. 3A). In SHSY-5Y cells with endogenous PrP, transfection of PrP-specific siRNA expressing plasmids did not result in a reduction of cell viabilities $12 \mathrm{~h}$ post-challenge, however, transfection of Ri3 expressing plasmid led to a significantly lower cell viability (Fig. 3B). To address whether knockdown of cellular PrP influences the cytotoxicities induced by other chemicals, cells were challenged with different amounts of $\mathrm{H}_{2} \mathrm{O}_{2}$ or colchicine, a microtubule-disrupting agent. No differences in cell viabilities between the groups receiving plasmids expressing PrP siRNAs and that of controls were seen (data not shown). The results suggest that the presence of PrP-specific siRNAs reduces the protective activity of cellular $\mathrm{PrP}$ against the challenge of copper.

Expression of PrP-specific siRNA partially antagonizes the cytotoxicity of fCJD-associated PrP mutants. Our previous studies have confirmed that expression of some familial CJD (fCJD)-associated PrP mutants with extra octarepeats can induce cytotoxicities in vitro (29). Prior to evaluation of the possible reversing effects of PrP siRNAs on the cytotoxicity induced by fCJD mutants, the inhibition of PrP siRNAs on the expression of the PrP mutants in the cells transfected with
PrP-PG9 and PrP-PG12 were assessed by Western blot analyses . Both Ri2 and Ri3 showed obvious repressive effects on the expression of PrP-PG9 and PrP-PG12 in SHSY-5Y and 293T cells (Fig. 4). Transfection of Ri3null did not change the expression levels of PrP-PG9 and PrP-PG12, while transfection of Ri2null, slightly but clearly repressed the expression levels of these two PrP mutants (Fig. 4).

SHSY-5Y and 293T cells were transiently transfected with plasmids expressing PrP-PG5, -PG9 and -PG12. As expected, the MTT assay showed clear reductions in the cell viabilities of the cells expressing PrP-PG9 and PrP-PG12 compared with those expressing PrP-PG5 and those transfected with blank vector, with an average 17\% reduction in the preparation of PrP-PG9 and a 21\% reduction in that of PrP-PG12 (data not shown). To evaluate the effects of PrP siRNAs on the cytotoxicity of PrP mutants, both cell lines were co-transfected with PrP-PG9 or PrP-PG12, together with various PrP siRNA constructs, and the cell viability was measured by the MTT assay 12 and $24 \mathrm{~h}$ after transfection. Co-expressions of Ri2 and Ri3 resulted in significantly higher cell viabilities in 293T cells expressing PrP-PG12 at 12 and at $24 \mathrm{~h}$ post-transfection, as well as at $12 \mathrm{~h}$ post-transfection in those cells expressing PrP-PG9, whereas co-expressions of Ri2null and Ri3null did not induce a change (Fig. 5A). Higher OD values were observed in all experimental cells expressing PrP-PG9 $24 \mathrm{~h}$ post-transfection, but without significant differences in the OD values among the various groups (Fig. 5A). Similar alterations in the cell viabilities were also observed in the cell line SHSY-5Y, in that both $\mathrm{Ri} 2$ and $\mathrm{Ri} 3$ increased the cell viability in the cells expressing PrP-PG9 and PrP-PG12 $12 \mathrm{~h}$ after transfection, while only Ri3 increased the cell viability in the cells expressing PrP-PG9 and PrP-PG12 $24 \mathrm{~h}$ after transfection (Fig. 5B). The data imply that PrP-specific siRNAs are able to partially antagonize the cytotoxicities induced by the transient expression of fCJD-associated PrP mutants. 
A $293 \mathrm{~T}$

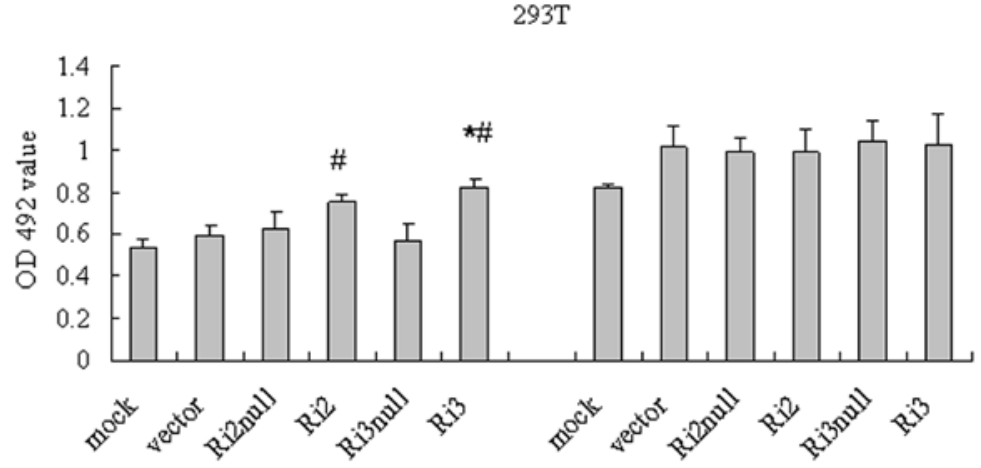

PrP-PG12

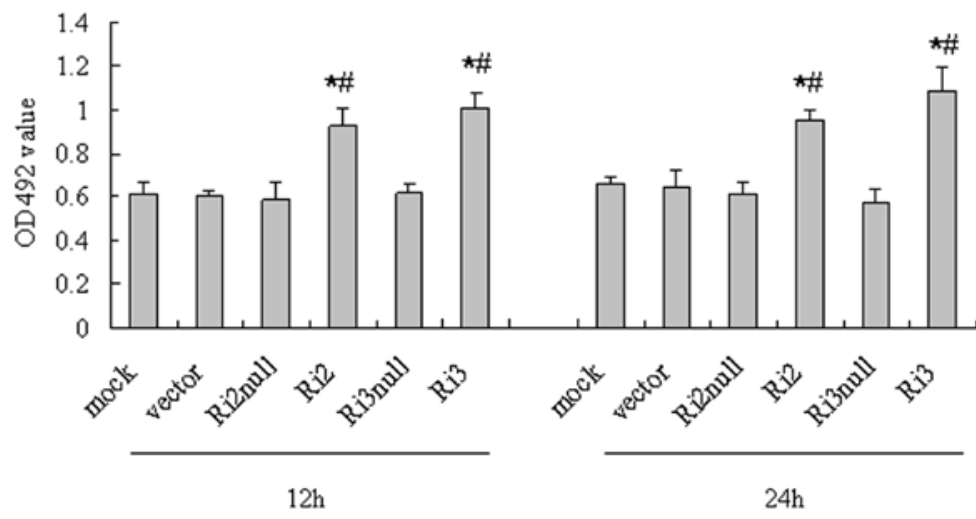

B
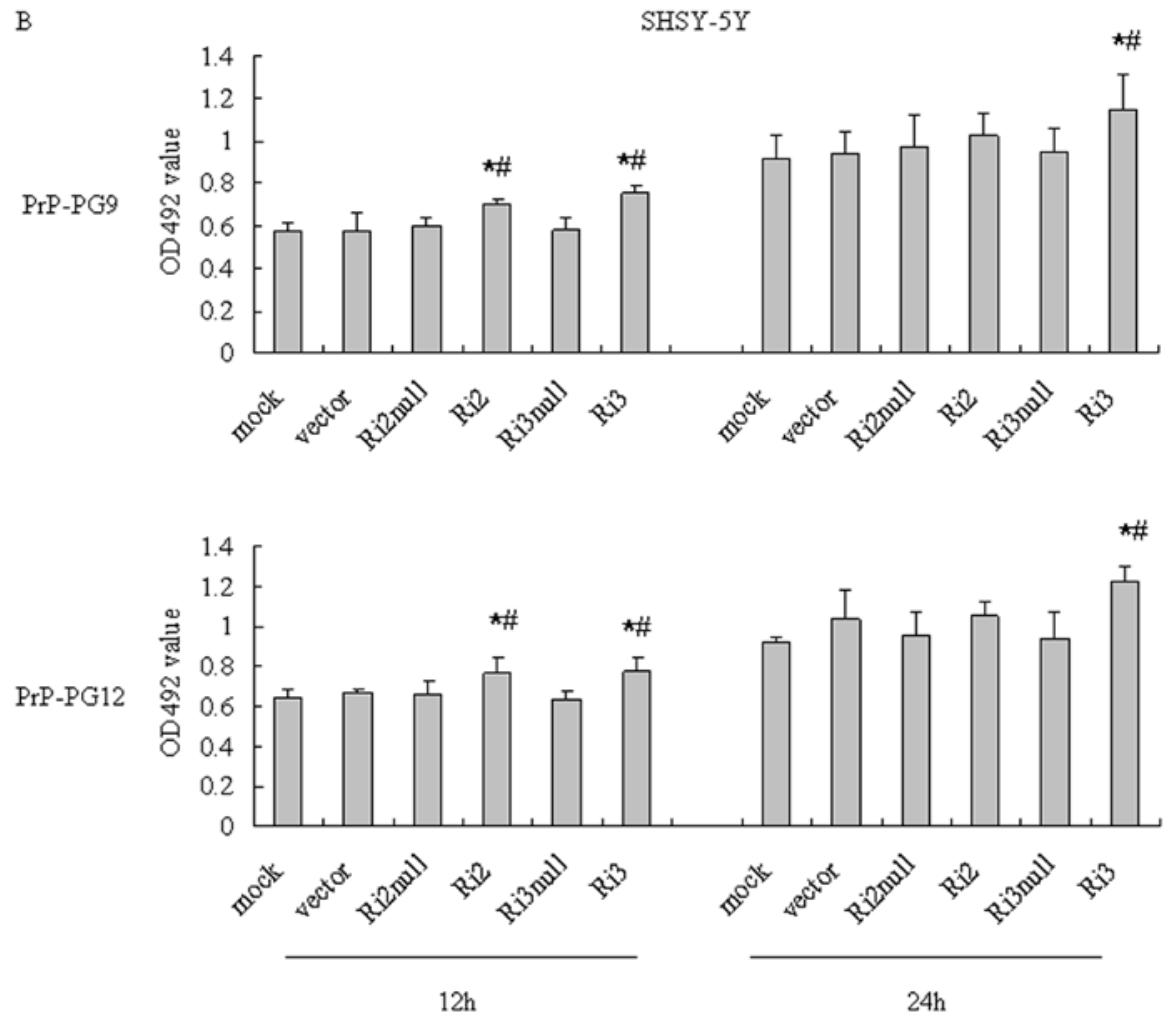

Figure 5. MTT assays for the antagonizing effects of Ri2 and Ri3 on the cytotoxicity induced by PrP-PG9 or PrP-PG12. Cells were co-transfected with PrPPG9 or PrP-PG12, together with various PrP siRNA constructs. The cell viability was measured by MTT assays $12 \mathrm{~h}$ (left panel) and $24 \mathrm{~h}$ (right panel) after transfection. (A) 293T cells. (B) SHSY-5Y cells. The average data of each preparation is calculated based on three independent experiments and represented

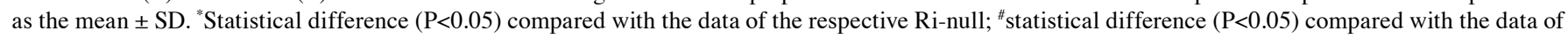
the mock control.

Expression of PrP-specific siRNA restores the cytotoxicity induced by the expression of fCJD-associated PrP mutants. To assess the effectiveness of the expressions of PrP-specific
siRNAs on the cytotoxicity caused by the expression of PrP mutants beforehand, cells received PrP-PG9 and PrP-PG12 expressing plasmids for 0,6 and $12 \mathrm{~h}$ were transfected with 


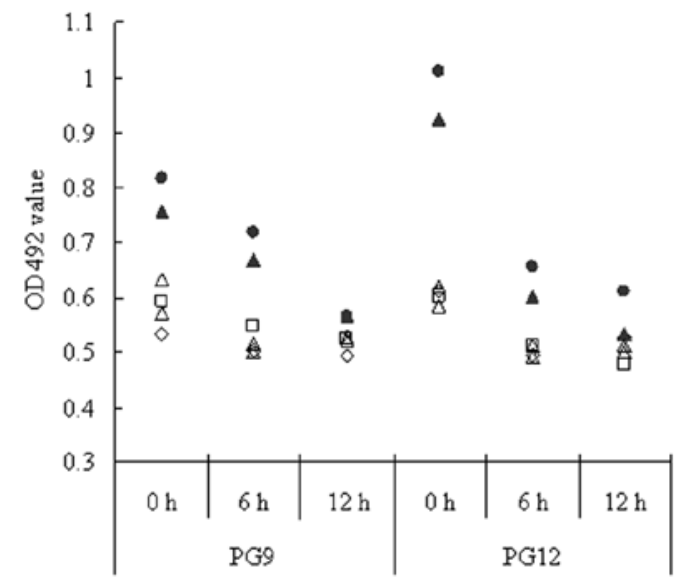

$293 \mathrm{~T}$

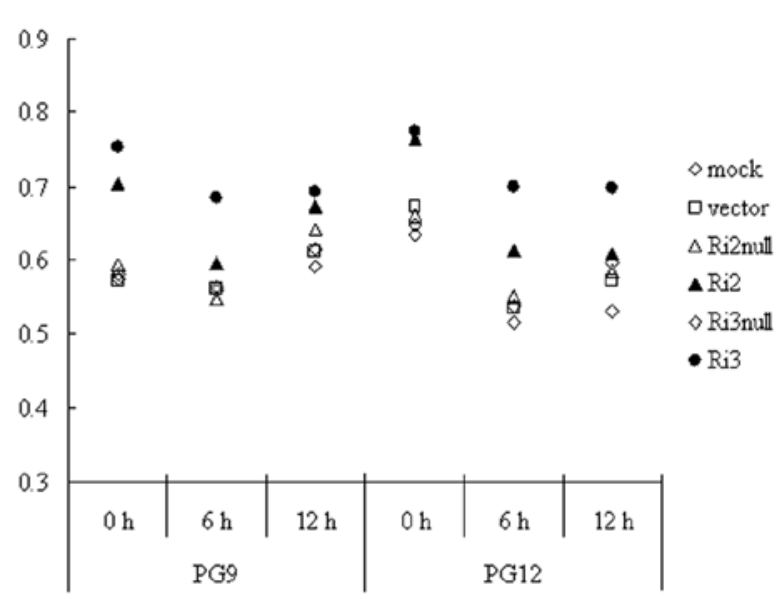

SHSY-5Y

Figure 6. Comparative analyses of the antagonizing activities of Ri2 and Ri3 on the cytotoxicity of PrP-PG9 or PrP-PG12 expressed 0, 6 and 12 h beforehand. Cell viability was measured by MTT assays $12 \mathrm{~h}$ after transfection of plasmids expressing PrP-specific siRNAs. (A) 293T cells. (B) SHSY-5Y cells. The average data of each preparation is calculated based on three independent experiments.

the plasmids expressing PrP-specific siRNAs and maintained for an additional $12 \mathrm{~h}$. The MTT assays showed that the OD values of $293 \mathrm{~T}$ cells expressing PrP-PG9 and PrP-PG12 decreased along with the maintaining times. Compared with that of mock, the viabilities of the cells transfected with blank vector, Ri2null and Ri3null for 0,6 and $12 \mathrm{~h}$ remained at comparably low levels (Fig. 6). In contrast, significantly higher OD values were observed in the cells expressing PrP-PG9 or PrP-PG12 for 0 and $6 \mathrm{~h}$ beforehand and subsequently transfected with Ri2 and Ri3, as well as that expressing PrP-PG12 for $12 \mathrm{~h}$ beforehand and subsequently transfected with Ri3 (Fig. 6). This highlights that the expression of PrP-specific siRNAs is able to restore the cytotoxicity induced by the presence of PrP mutants in advance. Compared with the observations in the cells expressing PrP-PG9 or PrP-PG12 for $6 \mathrm{~h}$, the restoring abilities of Ri2 and Ri3 were remarkably higher than that expressing PrP-PG9 or PrP-PG12 for $12 \mathrm{~h}$. These results suggest that the more efficient influences of PrP-specific siRNAs on the cytotoxicity of PrP mutants may occur at the early stage.

\section{Discussion}

Two human PrP-specific siRNAs have been constructed in this study, which show efficient inhibition of the expression of either endogenous or exogenous PrP in the cultured cell lines. The targeting sites of these siRNAs are located at aa 108-114 (Ri2) and aa 171-177 (Ri3), respectively. Introduction of these siRNAs into cells did not induce a detectable influence on cell growth. However, expression of PrP siRNAs obviously reduced the cell protective activity of wild-type PrP against the challenge of copper and partially antagonized the cytotoxicity of fCJD-associated PrP mutants.

$\mathrm{PrP}^{\mathrm{C}}$ can act as a copper ion buffer protecting cells from the effects of uncomplexed $\mathrm{Cu}^{2+}$ (30). Comparison between normal and PrP knockout mice has identified an extensive oxidative damage in knockout mice, but not in normal ones (31). $\mathrm{Cu}^{2+}$ induces cell apoptosis via multiple pathways, including damaging the structure of cell microtubules (32). Knockdown of the endogenous $\mathrm{PrP}^{\mathrm{C}}$ in SHSY-5Y cells or of the exogenously-induced wild-type PrP in 293T cells, by PrP-specific siRNAs in this report clearly reduced the resistance of the cells against the challenge of copper, indicating again that cellular $\operatorname{PrP}^{\mathrm{C}}$ is involved in not only the metabolism of copper, but also in the elimination of the side-effects of excessive $\mathrm{Cu}^{2+}$, so that neurons having wild-type PrP may be more resistant to copper toxicity than those lacking the protein $(20,33,34)$. In fact, based on hybrid DFT/DFT calculations different geometries and energetics of copper ion attachment to the PrP have been proposed according to different copper concentrations. At low concentrations, copper ion is bound to four histidine imidazole groups, whereas at higher concentrations, two histidine imidazoles, one of them in an axial position, coordinate the copper. Evaluation of the interplay between the number of bound ions and the binding strength has suggested that when enough copper is available, the binding site(s) will spontaneously be rearranged in order to accommodate more copper ions with a net energy gain, despite the fact that the binding strength per copper ion decreases with the number of ions per PrP. The strong affinity for copper and the multiplicity of concentration-dependent copper binding sites supports the suggested copper buffering role of the $\mathrm{PrP}$, as a storage place for excessive $\mathrm{Cu}^{2+}$ and protection of other proteins from the effects of uncomplexed copper (35).

The known fCJD-associated mutations are either point mutations located primarily at the C-terminal domain, or insertions of 1 to 9,12 or more extra octarepeats, resulting in the expansion of the N-terminal domain. Transgenic mice expressing insert mutants of PrP develop prion disease with accumulation of detergent-insoluble, protease-resistant PrP in the brain. When expressed in cell lines, PrP with additional repeats displays detergent insolubility, resistance to proteinase $\mathrm{K}$ digestion similar to $\mathrm{PrP}^{\mathrm{Sc}}$, and alters cell surface expression and hinders export to the cell surface, leading to obvious cytotoxicity $(24,36)$. The significant antagonizing activity of PrP-specific siRNAs on the octarepeats insertion-induced 
cytotoxicity illustrates not only the molecular mechanism of the mutants, but also the potential to use those siRNAs for eliminating the relevant neurotoxicity.

The onset time and progression of the fCJD with octarepeat expansion is associated with the numbers of inserts (37). Our previous studies have also revealed that the cytotoxicity is closely related with the numbers of the octarepeats $(24,38)$. More intensive cytotoxicity, regardless of reducing level or persistent time, has also been observed in the preparation of PrP-PG12 than in that of PrP-PG9 in this study. This may explain the observations that the rescuing activities of two PrP-specific siRNAs on PrP-PG12-induced cytotoxicity are relatively stronger than that of the PrP-PG9-induced one. This phenomenon may relate with the more active cytotoxicity of PrP-PG12. We have also noticed that the rescuing capacities of PrP-specific siRNAs are related with their administrating times; that the earlier the siRNAs are applied into the cells, the more efficient their interferences are. This further suggests that PrP-specific siRNAs can efficiently block the cytotoxicity caused by expressing PrP mutants de novo or slow the cytotoxicity contributed by constantly expressing PrP mutants, but have little effect on the already occurred cytotoxicity.

Under our experimental conditions, PrP-specific siRNA $\mathrm{Ri} 3$ repeatedly showed more active interference in either the protection of wild-type PrP against copper or the cytotoxicity of the PrP mutants with the extra octarepeats compared to Ri2. Both siRNAs target 18 nucleotides of PrP mRNA. The targeting sequence of Ri2 is located at aa 108-114 that is at the center of PrP106-126, and that of Ri3 is located at aa 171-177 covering the $\mathrm{N}$-terminus of the second $\alpha$-helix. Our data illustrate that Ri3 has stronger inhibitory effects on the PrP expression in both tested cell lines compared to Ri2, which may contribute to the functional differences of two siRNAs.

Although several anti-prion compounds have been reported to be effective in cell culture, no pharmaceutical treatment for TSEs is yet available $(8,39)$. There is great hope that novel therapeutical tools, including gene-based therapies, will be developed. Studies of RNAi-based therapies for neurodegenerative disorders are currently based on transgenic animal models that express disease-causing genes, e.g. polyglutamine induced neurodegeneration $(40,41)$ and Alzheimer's disease (42). This is quite different from a realistic therapeutic setting in humans, in which the siRNA can reduce the expression of both the diseased and normal allele, leading to unwanted side effects. Certainly, some unsolved problems, e.g. the potential activation of the innate immune system of dsRNAs, inhibition of non-target genes and saturation of RNAi machinery, still hamper the usage of the RNAi-based tool (43). In addition, safe and efficient delivery system for dsRNAs is in high demand. Studies on PrP-specific siRNAs will provide a useful tool for understanding the pathogenesis of prion disease and offer helpful clues for seeking potential therapy of prion disease in the future.

\section{Acknowledgements}

This study was supported by the China Mega-Project for Infectious Disease (2009ZX10004-101, 2008ZX10004008), a SKLID Development Grant (2008SKLID102), the National Basic Research Program of China (973 Program)
(2007CB310505), the Chinese National Natural Science Foundation Grants 30771914 and 30800975, and Institution Technique R\&D Grant (2008EG150300).

\section{References}

1. Prusiner SB: Prions. Proc Natl Acad Sci USA 95: 13363-13383, 1998.

2. Caughey B and Chesebro B: Prion protein and the transmissible spongiform encephalopathies. Trends Cell Biol 7: 56-62, 1997.

3. Griffith JS: Self-replication and scrapie. Nature 215: 1043-1044, 1967.

4. Bolton DC, McKinley MP and Prusiner SB: Identification of a protein that purifies with the scrapie prion. Science 218: 1309-1311, 1982.

5. McKinley MP, Bolton DC and Prusiner SB: A protease-resistant protein is a structural component of the scrapie prion. Cell 35: 57-62, 1983.

6. Bieschke J, Weber P, Sarafoff N, Beekes M, Giese A and Kretzschmar H: Autocatalytic self-propagation of misfolded prion protein. Proc Natl Acad Sci USA 101: 12207-12211, 2004.

7. Aguzzi A, Glatzel M, Montrasio F, Prinz M and Heppner FL: Interventional strategies against prion diseases. Nat Rev Neurosci 2: 745-749, 2001.

8. Trevitt CR and Collinge J: A systematic review of prion therapeutics in experimental models. Brain 129: 2241-2265, 2006.

9. Bueler H, Aguzzi A, Sailer A, et al: Mice devoid of PrP are resistant to scrapie. Cell 73: 1339-1347, 1993.

10. Sailer A, Bueler H, Fischer M, Aguzzi A and Weissmann C: No propagation of prions in mice devoid of PrP. Cell 77: 967-968, 1994.

11. Manson JC, Clarke AR, McBride PA, McConnell I and Hope J: PrP gene dosage determines the timing but not the final intensity or distribution of lesions in scrapie pathology. Neurodegeneration 3: 331-340, 1994

12. Mallucci G, Dickinson A, Linehan J, Klohn PC, Brandner S and Collinge J: Depleting neuronal PrP in prion infection prevents disease and reverses spongiosis. Science 302: 871-874, 2003.

13. Sen GL and Blau HM: A brief history of RNAi: the silence of the genes. FASEB J 20: 1293-1299, 2006.

14. Hammond SM, Bernstein E, Beach D and Hannon GJ: An RNA-directed nuclease mediates post-transcriptional gene silencing in Drosophila cells. Nature 404: 293-296, 2000.

15. Pfeifer A, Eigenbrod S, Al-Khadra S, et al: Lentivector-mediated RNAi efficiently suppresses prion protein and prolongs survival of scrapie-infected mice. J Clin Invest 116: 3204-3210, 2006.

16. White MD, Farmer M, Mirabile I, Brandner S, Collinge J and Mallucci GR: Single treatment with RNAi against prion protein rescues early neuronal dysfunction and prolongs survival in mice with prion disease. Proc Natl Acad Sci USA 105: 10238-10243, 2008.

17. Kim Y, Han B, Titlow W, Mays CE, Kwon M and Ryou C: Utility of RNAi-mediated prnp gene silencing in neuroblastoma cells permanently infected by prions: potentials and limitations. Antiviral Res 84: 185-193, 2009.

18. Millhauser GL: Copper binding in the prion protein. Acc Chem Res 37: 79-85, 2004.

19. Millhauser GL: Copper and the prion protein: methods, structures, function, and disease. Annu Rev Phys Chem 58: 299-320, 2007.

20. Vassallo N and Herms J: Cellular prion protein function in copper homeostasis and redox signalling at the synapse. J Neurochem 86: 538-544, 2003.

21. Weissmann $C$ and Flechsig E: PrP knock-out and PrP transgenic mice in prion research. Br Med Bull 66: 43-60, 2003.

22. Brown DR: Role of the prion protein in copper turnover in astrocytes. Neurobiol Dis 15: 534-543, 2004.

23. Kubosaki A, Nishimura-Nasu Y, Nishimura T, et al: Expression of normal cellular prion protein $(\operatorname{PrP}(\mathrm{c}))$ on $\mathrm{T}$ lymphocytes and the effect of copper ion: analysis by wild-type and prion protein genedeficient mice. Biochem Biophys Res Commun 307: 810-813, 2003.

24. Bizat N, Peyrin JM, Haik S, et al: Neuron dysfunction is induced by prion protein with an insertional mutation via a Fyn kinase and reversed by sirtuin activation in Caenorhabditis elegans. $\mathrm{J}$ Neurosci 30: 5394-5403, 2010.

25. Campana V, Sarnataro D, Fasano C, Casanova P, Paladino S and Zurzolo C: Detergent-resistant membrane domains but not the proteasome are involved in the misfolding of a PrP mutant retained in the endoplasmic reticulum. J Cell Sci 119: 433-442, 2006. 
26. Chen L, Yang Y, Han J, et al: Removal of the glycosylation of prion protein provokes apoptosis in SF126. J Biochem Mol Biol 40: 662-669, 2007.

27. An R, Dong C, Lei Y, et al: PrP mutants with different numbers of octarepeat sequences are more susceptible to the oxidative stress. Sci China C Life Sci 51: 630-639, 2008

28. Rachidi W, Riondel J, McMahon HM and Favier A: Prion protein and copper: a mysterious relationship. Pathol Biol (Paris) 53: 244-250, 2005 (In French).

29. Dong CF, Shi S, Wang XF, et al: The N-terminus of PrP is responsible for interacting with tubulin and fCJD related PrP mutants possess stronger inhibitive effect on microtubule assembly in vitro. Arch Biochem Biophys 470: 83-92, 2008.

30. Brown DR, Qin K, Herms JW, et al: The cellular prion protein binds copper in vivo. Nature 390: 684-687, 1997.

31. Klamt F, Dal-Pizzol F, Conte da Frota ML Jr, et al: Imbalance of antioxidant defense in mice lacking cellular prion protein. Free Radic Biol Med 30: 1137-1144, 2001.

32. Raes H, Braeckman BP, Criel GR, Rzeznik U and Vanfleteren JR Copper induces apoptosis in Aedes C6/36 cells. J Exp Zool 286 $1-12,2000$

33. Rachidi W, Vilette D, Guiraud P, et al: Expression of prion protein increases cellular copper binding and antioxidant enzyme activities but not copper delivery. J Biol Chem 278: 9064-9072, 2003.

34. Zeng F, Watt NT, Walmsley AR and Hooper NM: Tethering the $\mathrm{N}$-terminus of the prion protein compromises the cellular response to oxidative stress. J Neurochem 84: 480-490, 2003.
35. Hodak M, Chisnell R, Lu W and Bernholc J: Functional implications of multistage copper binding to the prion protein. Proc Natl Acad Sci USA 106: 11576-11581, 2009.

36. Solomon IH, Huettner JE and Harris DA: Neurotoxic mutants of the prion protein induce spontaneous ionic currents in cultured cells. J Biol Chem 285: 26719-26726, 2010.

37. Stevens DJ, Walter ED, Rodriguez A, et al: Early onset prion disease from octarepeat expansion correlates with copper binding properties. PLoS Pathog 5: e1000390, 2009.

38. Li XL, Wang GR, Jing YY, et al: Cytosolic PrP induces apoptosis of cell by disrupting microtubule assembly. J Mol Neurosci 43: 316-325, 2011.

39. Aguzzi A, Heikenwalder M and Miele G: Progress and problems in the biology, diagnostics, and therapeutics of prion diseases. $\mathrm{J}$ Clin Invest 114: 153-160, 2004.

40. Harper SQ, Staber PD, He X, et al: RNA interference improves motor and neuropathological abnormalities in a Huntington's disease mouse model. Proc Natl Acad Sci USA 102: 5820-5825, 2005.

41. Xia H, Mao Q, Eliason SL, et al: RNAi suppresses polyglutamineinduced neurodegeneration in a model of spinocerebellar ataxia. Nat Med 10: 816-820, 2004.

42. Singer O, Marr RA, Rockenstein E, et al: Targeting BACE1 with siRNAs ameliorates Alzheimer disease neuropathology in a transgenic model. Nat Neurosci 8: 1343-1349, 2005.

43. Chowdhury D and Novina CD: RNAi and RNA-based regulation of immune system function. Adv Immunol 88: 267-292, 2005. 